\title{
THE EFFECTS OF COHERENCE CALCULATION ON FOREST HEIGHT ESTIMATION USING SINC MODEL
}

\author{
Zhe Wen ${ }^{1,2}$, Lei Zhao ${ }^{1, *}$, Wangfei Zhang ${ }^{2}$, Erxue Chen $^{1}$, Kunpeng $\mathrm{Xu}^{1}$ \\ ${ }^{1}$ IFRIT, Chinese Academy of Forestry, Xiangshan road, Beijing, China - zhaolei@ifrit.ac.cn \\ ${ }^{2}$ College of Forestry, Southwest Forestry University, Kunming, China - mewhff@163.com
}

KEY WORDS: Coherence, SINC model, Forest height, Calculation algorithms, Window size

\begin{abstract}
:
In this paper, the effects of coherence on forest height estimation by SINC model based on Tandem-X InSAR data were explored. First, different coherence calculation methods and different window sizes were used to obtain interferometric coherence images. Then, the forest heights were obtained based on SINC model. Finally, the estimated forest heights were validated against reference data from airborne LIDAR CHM (Canopy height model, CHM). The results showed that the coherence calculation algorithm affect the forest height inversion results with SINC model. The algorithm using only phase information for coherence calculation show better performance than the other one using both magnitude and phase information. Meanwhile, window size selecting for coherence calculation also affect the forest height estimation results. In this study, window size with $9 \times 9$ shows best agreement with the forest height extracted from LiDAR CHM. the $\mathrm{R}^{2}$ and RMSE are 0.656 and $3.54 \mathrm{~m}$, respectively.
\end{abstract}

\section{INTRODUCTION}

Interferometric synthetic aperture Radar (InSAR) technology has the potential to extract the vertical structure information of the target. It also shows a great potential to get forest height in large area. For this reason, it attracts lots of interest of scholars in this field. There are two dominant methods of forest height estimation using InSAR technology. One method is to perform interference processing on the original data to obtain the DSM(Digital Surface Model, DSM), and then use the DSM minus DEM(Digital Elevation Model, DEM) to obtain the forest height. The other is based on coherent scattering model and coherence information to forest height inversion. The former needs the supporting of high-resolution DEM, which limits its use in large areas. The latter one has the advantage of no needing of high-resolution DEM data. Among the methods with coherent scattering model and coherence information, SINC model was approved a simple and reliable method for forest height inversion (Cloude, 2016). It is simplified from the RVoG (Random Volume over Ground) model. In the SINC model, two parameter including vertical wave number $(\mathrm{kz})$ and the values of coherence coefficients affect the estimation results. For the influence of $\mathrm{kz}$, a lot of research has been carried out on its effects (Chen, 2016; Kugler, 2015). However, the effects of coherence have not been fully explored yet (Hashjin, 2013). In this paper, we aim to explore the effects of coherence on forest height inversion with SINC model. The influence on coherence from the algorithms for the calculation of coherence values and the window size selected for coherence calculation were analyzed in this paper. We also explored the effect of the flat earth on forest height estimated by SINC model.

\section{STUDY AREA AND DATA}

\subsection{Study area}

The test site is located in GenHe, Inner Mongolia, China $\left(121.5^{\circ} \mathrm{E}, 50.8^{\circ} \mathrm{N}\right)$. Forest covered $91.7 \%$ of the test site and the area is .745 million hectares. The dominant tree species includes Xin`an Larch(Larix gmelinii(Rupr)Kuzen)、Sylvestris(Pinus sylvestris var. mongolica Litv.)、White birch(Betula platyphylla Suk.)and Aspen(Populus davidiana Dode). Figure 1 shows the location of the test site and the coverage of the acquired TanDEM-X data(inside the blue box) and LiDAR data(inside the red box) which used for forest height inversion and results validation in the test site, respectively.

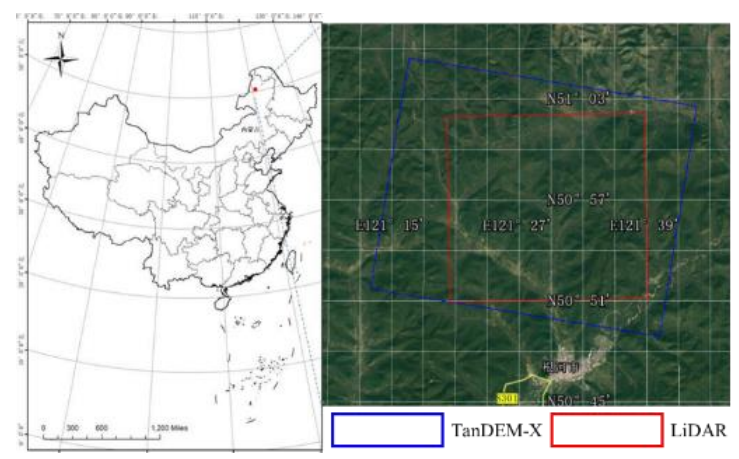

Figure 1. Scope of study area and range of remote sensing data

\footnotetext{
* Corresponding author
} 


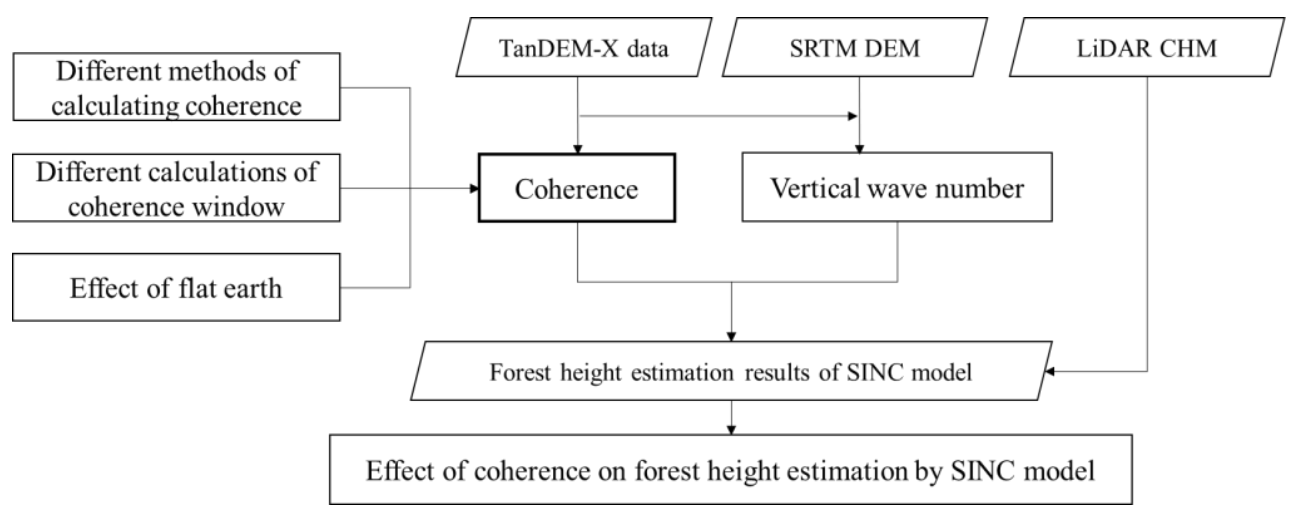

Figure 2. The flowchart of this study

\subsection{Remote sensing data}

\subsubsection{TanDEM-X}

In this study, we chose TanDEM-X data as experimental data. Due to the special satellite system, it has no temporal decorrelation, so it has high coherence, which is very helpful for our research. We use a TanDEM-X strip mode map image pair acquired on February 28, 2014. The baseline length is $138.48 \mathrm{~m}$, and the central of incidence angle is $42.21^{\circ}$. The height of ambiguity is $53.92 \mathrm{~m}$. The pixel spacing of range direction and azimuth direction are 1.37 and $2.84 \mathrm{~m}$, respectively.

\subsubsection{SRTM DEM}

The $30 \mathrm{~m} \times 30 \mathrm{~m}$ resolution SRTM (Shuttle Radar Topography Mission, SRTM) DEM data was used in this study as terrain reference. It was also used to calculate the local incident angle, and further the parameter $\mathrm{kz}$ can be calculated. The parameter $\mathrm{kz}$ was required for forest height estimation using SINC model.

\subsubsection{LiDAR CHM}

LiDAR data was applied to validate the forest height estimated from TanDEM-X data based on SINC model. The acquisition time of LiDAR data was August 2012. The "Yun-5" aircraft was used as the platform and the Leica LiDAR system was used for flight tests. According to experience, the mean dominant height tends to have a better correlation between forest height results estimated by SINC model. So the mean dominant height was chosen to validate the estimation result. It is defined as the average height of the highest 100 trees per hectare in the forest covered area (Aire, 2016; Aulinger, 2005). The mean dominant height of LiDAR data was extracted by a $10 \mathrm{~m} \times 10 \mathrm{~m}$ window of maximum filtering method from original LiDAR CHM data with $1 \mathrm{~m}$ resolution. We define the processed data as LiDAR CHM H100. Afterwards, the processed LiDAR CHM H100 and SINC model estimation results are resampled to the same resolution for accuracy verification.

\section{METHOD}

Figure 2 is the flowchart of this study. First, coherence was calculated from TanDEM-X image pairs. Second, $k z$ file was extracted based on the satellite parameter file and the terrain reference file. LiDAR data will be used to evaluate the forest height results of each coherence calculation.

$$
k z=\frac{4 \pi B_{n}}{\lambda} \frac{1}{R \sin \theta}
$$

Equation (1) was used for calculation of the values of $k z$, Where $B_{n}$ is the vertical baseline, $R$ is the distance between the satellite and the target, $\theta$ is the central incidence angle of the TanDEM$\mathrm{X}$ images.

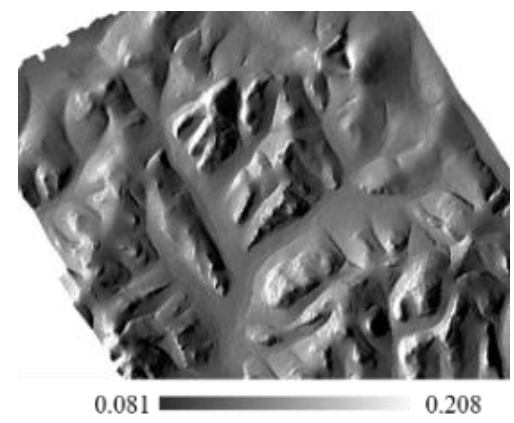

Figure 3. Vertical wave number

Figure 3 shows the image of calculated $k z$ file., Equation (2) describes the SINC model used for forest height estimation. $k z$ values were calculated from equation(1). $h_{v}$ represents forest height,$\gamma_{V}$ is the volume decorrelation of forest area.

$$
h_{v} \approx \frac{2 \pi}{k_{z}}\left(1-\frac{2}{\pi} \sin ^{-1}\left(\left|\gamma_{V}\right|^{0.8}\right)\right)
$$

In the experiment, we corrected the coherence when using the SINC model to calculate forest height. The most we concerned and most desired is volume decorrelation. In the interference processing of TanDEM-X CoSSC data, we need to consider signal-to-noise ratio(SNR) decorrelation. Equation (3) shows the calculation process of SNR decorrelation.

$$
\gamma_{S N R}=\frac{1}{1+(\mathrm{SNR})^{-1}}=\frac{1}{\sqrt{\left(1+\mathrm{SNR}_{1}^{-1}\right)\left(1+\mathrm{SNR}_{2}^{-1}\right)}}
$$

We can get the noise level of the SLC from TanDEM-X metadata. $\mathrm{SNR}_{1}$ and $\mathrm{SNR}_{2}$ represent the noise level of the master and slave images, respectively. Using the $\gamma_{S N R}$ obtained by equation (3), we can correct the coherence by equation (4)

$$
\gamma_{V}=\frac{\gamma}{\gamma_{S N R}}
$$

Since there is no need to consider temporal decorrelation and other decorrelations, we think the coherence obtained at this time is close to volume decorrelation. 


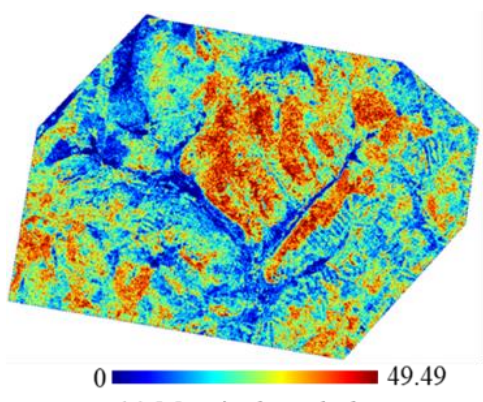

(a) Magnitude and phase

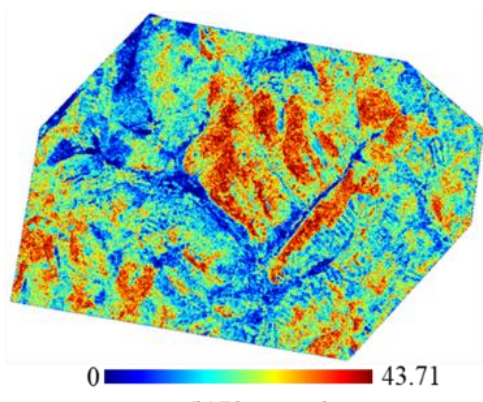

(b)Phase only

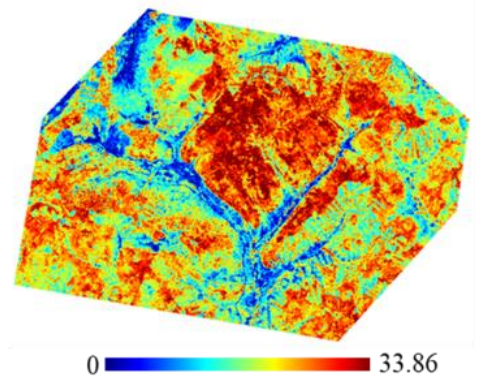

(c)LiDAR CHM H100

Figure 4. Estimation of forest height with two different coherence calculation algorithms
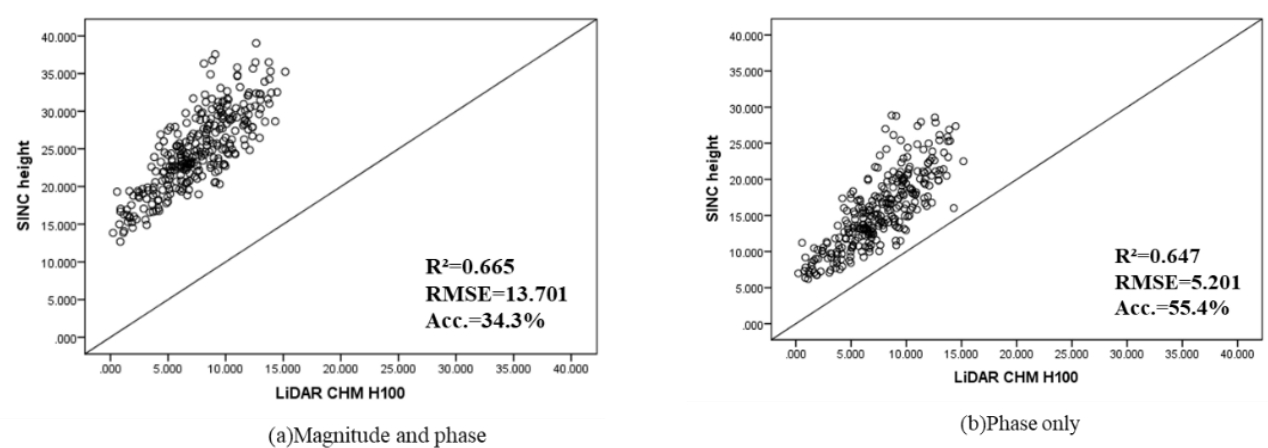

Figure 5. Forest height accuracy verification with different coherence estimation methods

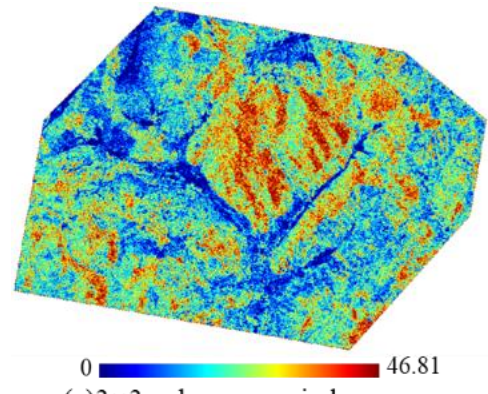

(a) $3 \times 3$ coherence window

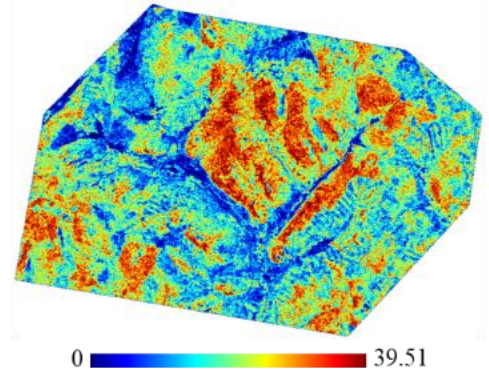

(b) $9 \times 9$ coherence window

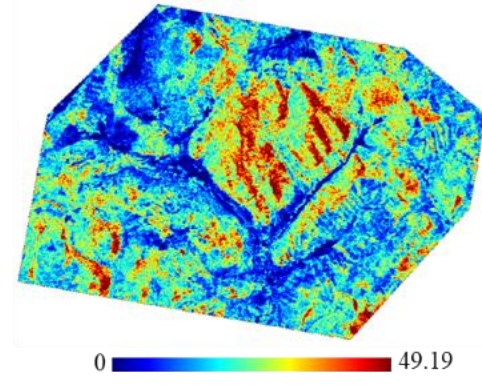

(c) $15 \times 15$ coherence window

Figure 6. Forest height estimation results with different window sizes

\subsection{Coherence calculation algorithms}

The coherence of two signals is defined as Equation (5):

$$
\gamma=\frac{\left|E\left[u_{1} \cdot u_{2}^{*}\right]\right|}{\sqrt{E\left[\left|u_{1}\right|^{2}\right] E\left[\left|u_{2}\right|^{2}\right]}}
$$

Equation (6) describes the coherence estimation process,

$$
\gamma=\frac{\left|\sum_{n=1}^{N} \sum_{m=1}^{M} u_{1}(n, m) \cdot u_{2}^{*}(n, m)\right|}{\sqrt{\sum_{n=1}^{N} \sum_{m=1}^{M}\left|u_{1}(n, m)\right|^{2}\left|u_{2}(n, m)\right|^{2}}}
$$

Where $\gamma$ is the coherence, $N$ and $M$ represent the estimated window size, respectively, $u_{1}$ and $u_{2}$ denote the pixel pairs in interference image pairs. Two methods of coherence calculation were used in this paper. The first one is to calculate the coherence with both phase and magnitude information in the two interferometric images. The second is to calculate the coherence only with phase information but assume the magnitude as 1 or a constant.

\subsection{Window size selecting for coherence calculation}

From equation (6), we can see that window size also affects the values of calculated coherence. Then the results of calculated coherence will in turn affect the forest height estimation results by SINC model. To find the effects of window size, we selected different window sizes for coherence calculating and then used the SINC model to estimate the related forest height.

\subsection{Effect of flat earth}

Base on formula of coherence calculation, when calculating coherence, whether to remove the flat earth influences the result of coherence calculation. We use the coherence obtained by removing the flat earth and the coherence obtained by not removing flat earth to estimate the forest height with SINC model and evaluate the results of both. 

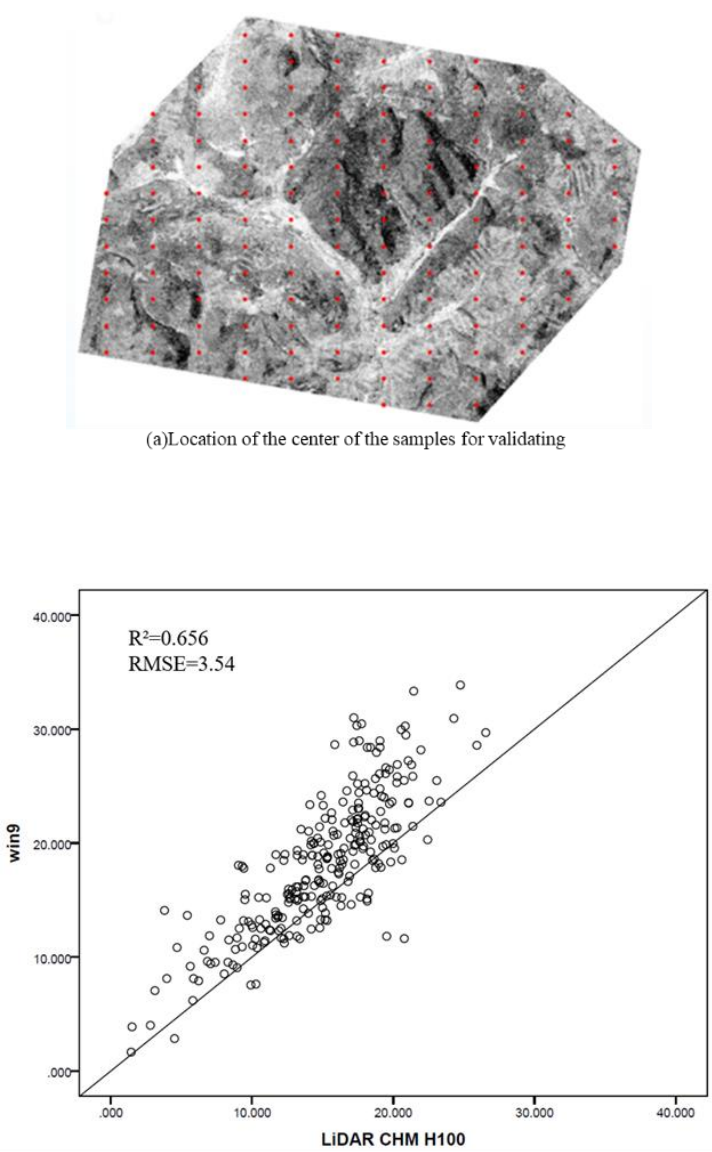

(c) $9 \times 9$ coherence window

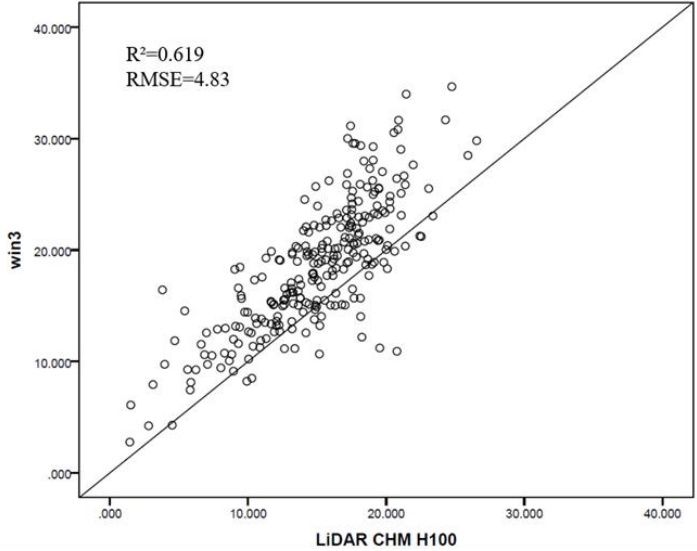

(b) $3 \times 3$ coherence window

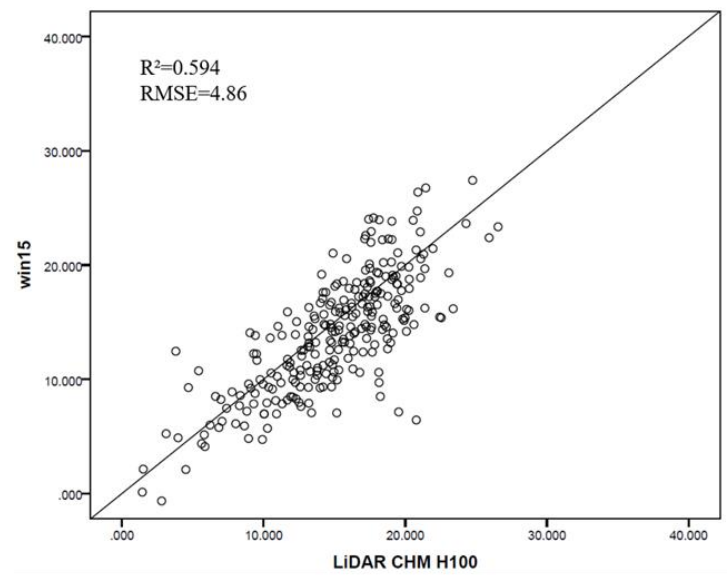

(d) $15 \times 15$ coherence window

Figure 7. Validation of estimated forest height with different window size

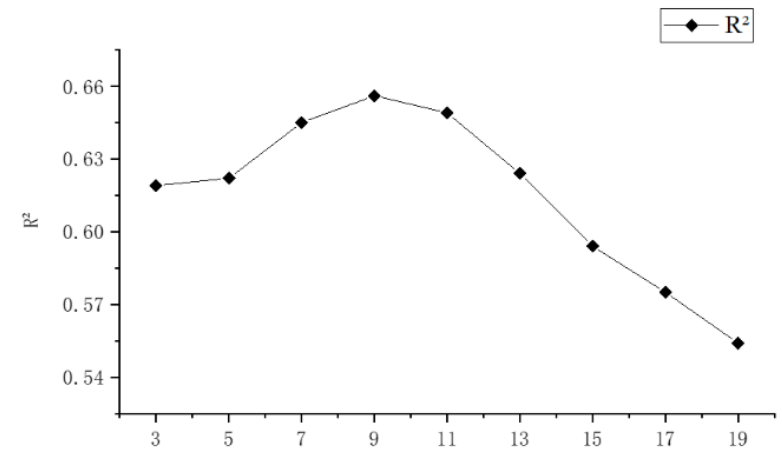

Figure 8 . The relationship between $\mathrm{R}^{2}$ and the sizes of window

\section{RESULTS AND ANALYSIS}

\subsection{Forest height from different coherence estimation}

\section{methods}

Figure 4 shows the results from two different coherence calculating algorithms, Figure 4(a) for the method using both magnitude and phase, Figure 4(b) for the method with only phase

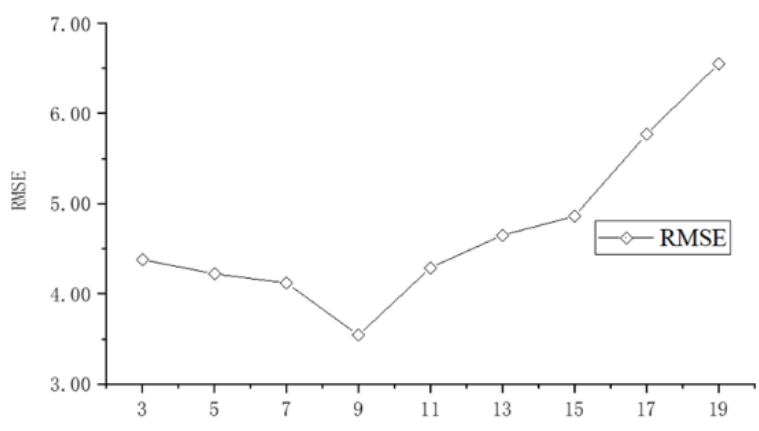

Figure 9. The relationship between RMSE and the sizes of window

information. The selected window size here was $5 \times 5$. 200 verification samples were randomly selected to further compare and analyze the estimation results of the two methods. Figure 5 shows the comparison of the forest height estimation results with coherence calculated from two different algorithms. It revealed that coherence calculation algorithms affect the forest height estimation results with SINC model. Moreover, the coherence calculated only with the phase information show better performance in forest height inversion. The value of the forest height estimated in higher accuracy. 


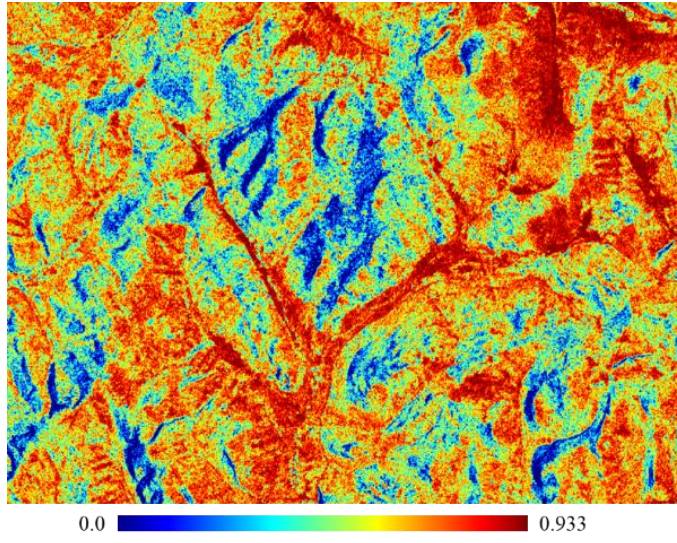

(a)Flat earth not remove

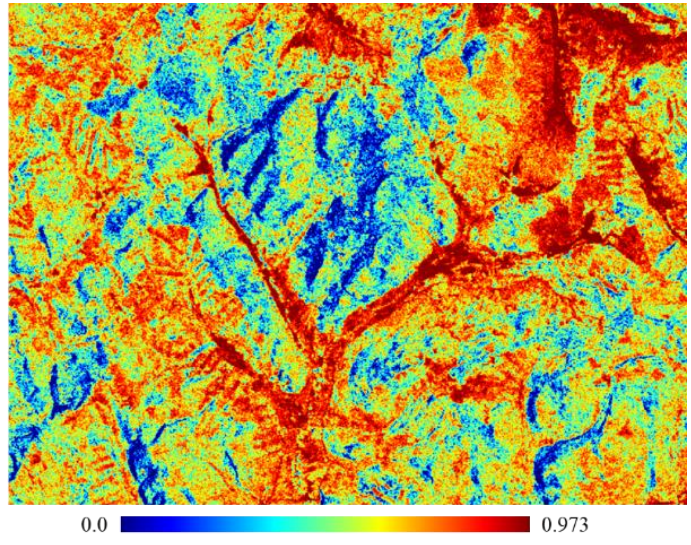

(b) Flat earth remove

Figure 10. The effect of flat earth on coherence

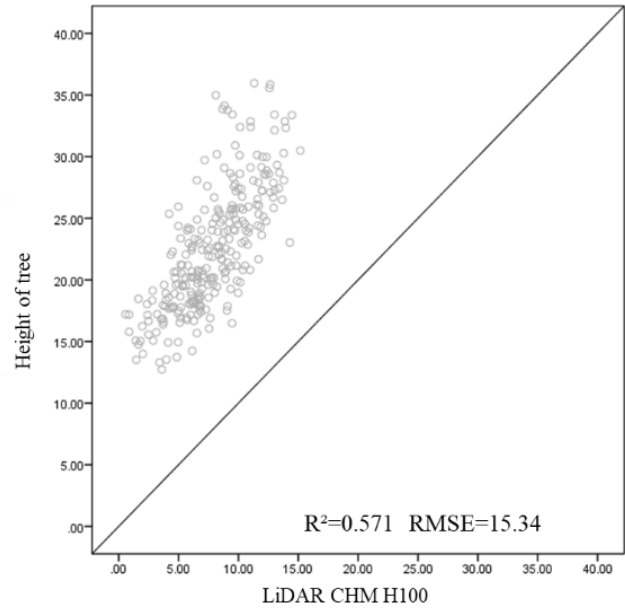

(a) Result of flat earth not remove

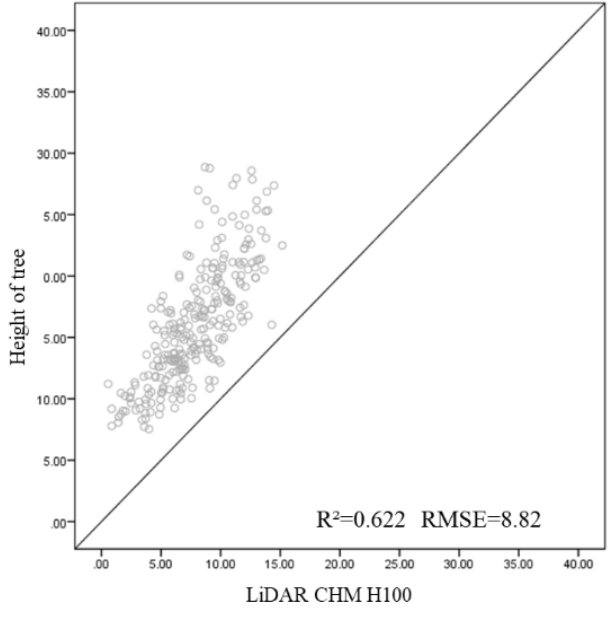

(b) Result of flat earth remove

Figure 11. Influence of flat earth on forest height estimation by SINC model

\subsection{Estimation of forest height for different window sizes}

The research in this section uses a coherence estimation method that considers only phase, Coherences were estimated using 9 different windows size. The window size included $3 \times 3,5 \times 5$, $7 \times 7 \ldots, 17 \times 17,19 \times 19$, respectively. The forest height estimation results with window size of $3 \times 3,9 \times 9,15 \times 15$ were selected as examples showed in Figures 6(a), (b), and (c).respectively. 200 sample points were selected for the estimation results validation. Figure 7 (a) shows the distribution of the samples. The values of $\mathrm{R}^{2}$ and RMSE between the estimated forest height and the height from LiDAR CHM with different window size are shown in Figure 7 (b),(c),(d). Figure 8 shows the relationship between the $\mathrm{R}^{2}$ values and the sizes of window selecting for coherence calculation, and Figure9 is the relationship between RMSE and the sizes of windows. They showed that window size of $9 \times 9$ has the best performance in forest height estimation by SINC model.

\subsection{Whether to remove the flat earth}

We use the method that only considers the phase to calculate the coherence. The coherence was calculated by the two methods of removing the flat earth and not removing the flat earth, as shown in Figure 10. We can see that the coherence in Figure 10 (b) is slightly higher than that in Figure 10 (a). We used two results to estimate the forest height by SINC model and evaluated them using the verification data. As shown in Figure 11, removing the flat earth can obtain better forest height estimation results. The forest height result obtained by removing the flat earth has an RMSE of 8.82. The forest height result without removing the flat earth has an RMSE of 15.34 .

This is because when calculating the coherence, if the phase is included flat earth, it will lead to an offset of the phase. The coherence obtained by not removing the flat earth will lower than that obtained by removing the flat earth.

\section{CONCLUSION}

In this study, difference coherence from calculation algorithm and window size selecting for samples were explored. Then their effects on SINC model for forest height inversion were analyzed. From the research, we conclude that the coherence calculation algorithm and the window size selecting in averaging of coherence calculation affect the forest height estimation results with SINC model. First, we found that for the SINC model, it is better to consider only the phase to calculate coherence. Second, there is an optimal window for coherence calculation. The 
optimal window for this paper is $9 \times 9$. We realize that we cannot blindly increase the size of the coherence calculation window. As we increase the window, the resolution of coherence will loss. Finally, we also discussed the effect of the flat earth on the forest height estimated by SINC model. We get better results with the flat earth removed. The next work is to explore how to select the window of coherence calculation quickly or even automatically.

\section{ACKNOWLEDGEMENTS}

This study was funded by the National Key R\&D Program of China (Grant No.2017YFB0502700), and the National Natural Science Foundation of China (Grant Nos. 41801289 and 31860240).

\section{REFERENCES}

Aire O, Jaan P, Oleg A, et al. 2016. Interferometric SAR Coherence Models for Characterization of Hemiboreal Forests Using TanDEM-X Data. Remote Sensing, 8(9):700-.

Aulinger T, Mette T, Papathanassion K P. et al. 2005. Validation of Heights from Interferometric SAR and LIDAR over the Temperate Forest Site "Nationalpark Bayerischer Wald". European Space Agency Esa Sp, 586:11.

Cloude S. 2010. Polarisation: applications in remote sensing. Oxford University Press, New York

Chen H , Cloude S R, Goodenough D G .2016. Forest Canopy Height Estimation Using Tandem-X Coherence Data. IEEE Journal of Selected Topics in Applied Earth Observations and Remote Sensing, 9(7):3177-3188.

Hashjin S S , Khazai S, Sadeghi A . 2013 A Method to Select Coherence Window Size for forest height estimation using PolInSAR Data. ISPRS.

Kugler F , Lee S K , Hajnsek I , et al. 2015, Forest Height Estimation by Means of Pol-InSAR Data Inversion: The Role of the Vertical Wavenumber. IEEE Transactions on Geoscience and Remote Sensing, 53(10):5294-5311. 